\title{
USE BIM TO CONSTRUCT ELECTRONIC RESUME SYSTEM FOR BUILDING PROJECT
}

\author{
$\underline{\text { Yan-Chyuan Shiau }}^{1 *}$, Ming-Teh Wang ${ }^{1}$, Chi-Huan Lin ${ }^{2}$, and Zhe-Yu Guo ${ }^{2}$ \\ ${ }^{1}$ Department of Construction Management, Chung Hua University, Hsin-Chu, Taiwan \\ ${ }^{2}$ Master Degree, Department of Construction Management, Chung Hua University, Hsin-Chu, Taiwan \\ *Corresponding author (ycshiau@ms22.hinet.net)
}

\begin{abstract}
The main function of Building Information Modeling is to establish the common internal project-related information for each stage for the whole life cycle of the building. User can fetch and store related information in this integrated platform. The data input by former user can be fetched by other relevant personnel to improve project quality, save time, as well as reduce costs and errors. The BIM system can precisely display visual result to achieve better communication. This can help engineers to perform better control to cost, schedule, and environmental impact. It can effectively display design details to engineers, builders and owners to improve communication effort to the whole team. This study uses BIM, ER Model, ASP.net, RFID, Database and Windows environment to develop "Electronic Building Construction Resume System". All projects are divided into Build, Floor, and Room units and assigned an RFID tag. All related data such as owner, design engineers, contractors, and inspectors are linked to this unique ID. Related documents such as specifications, drawings, pictures, films, checking list, and daily report are integrated and can be retrieved through ID. When the tag is detected by reader, one can connect to server and fetch related information to identify the responsibility for possible construction defects. This will push all participants realize the duties which are assigned to the project. In maintenance stage of the building, all construction details for each component can be fetched to verify the responsibility.
\end{abstract}

Keywords: Database, ER Model, Construction Resume, RFID, BIM

\section{INTRODUCTION}

\subsection{Research Background}

In recent years, a significant amount of effort has been invested in the synthesis of traditional industries and electronic technologies in order to boost the competitiveness of traditional industries, thereby assisting them to establish their presence in the international market. The construction industry in Taiwan has once been the leading sector among traditional industries and in order to achieve sustained operation, it has also adopted electronic technologies and digitization in recent years.

Generally speaking, the preservation of drawings and management of relevant documentations on pertinent regulations would not be much of an issue to the construction industry during the design and construction phases. However, in light of the fact that the use and maintenance of buildings usually last for decades and the management/access of design drawings for such lengthy durations would be a daunting task. Not to mention the difficulties involved in the validation of relevant subcontractors, construction workers and supervisors. And thus, the management of such documentations has become a significant shortcoming in terms of buildings' lifecycle management. It would therefore be very useful if one were to develop a management software that is capable of integrating all relevant drawings for construction, documentations, checklists and so forth in conjunction with the registration of construction personnel involved. The creation of such software would no doubt be very beneficial to the management of building lifecycles.

\subsection{Research motivation}

It should be of no surprise that building construction has a 
fairly long lifecycle with tedious and sophisticated paperwork at various different stages. The preservation of these documentations, such as preliminary bidding, subcontracting agreements, contracts, drawings at various design phases, exploded diagram of various parts, construction regulations, quality audit records, daily reports and maintenance records can be overwhelming and difficult to manage. Coupled with the fact that the scale and complexity of construction projects have been growing steadily over the years with more and more professional teams taking part in a single project, the task of identifying liabilities after construction has completed would be extremely difficult if flaws and defects during the construction were overlooked. And as such, in the pursuit for the improvement in construction quality and integration of architectural information, adequate management of construction procedural records and logs remains as an important issue that has to be addressed.

\subsection{Research objectives}

For the purpose of this research, tools such as RFID, ER Model, Building Information Modeling (BIM) system, database and so forth have been chosen to complement the "Electronic Building Construction Resume System", which would be developed in a web-based environment. The objectives of this research can therefore be summarized into the following points:

A. To review the current status of BIM and RFID usage in different industries through review of relevant literature and discuss system functions and database architecture.

B. To use the ER Model to create system architecture, including data tables, fields, attributes, PK, FK, and their correlation.

C. To discuss the parameters that come with objects in the BIM and propose solutions to incorporate the required fields in the database.

D. To develop the "Electronic Building Construction Resume System" software to facilitate the management of construction related resumes, including the personnel data of construction workers, subcontractor information, specifications, drawings, pictures, and video tapes.

E. To verify system functions by entering data from the case project and obtaining user feedback to ensure the functionality.

\section{ANDLYSIS OF CURRENT STATUS AND ISSUES}

Given the long lifecycles of construction projects, which involve planning, design, purchasing, subcontracting, construction, usage and maintenance of buildings; the sheer magnitude and costs involved in projects for the construction industry and the degree of sophistication involved in professional task division, the incorporation of CALS strategies and technologies to the overall construction process would seem fitting. The process would involve the digitization and standardization of key engineering information at each phase of a construction project's lifecycle so that all engineering data could be rapidly exchanged and shared through databases and the internet so as to lower relevant costs while ensuring quality and improving implementation efficiency. In addition, it would also facilitate economic and efficient use and maintenance of various construction facilities [1].

\subsection{Introduction of Electronic Resume System}

During the construction phase, building lifecycle would relate most directly to proprietors, designers and the party responsible for the construction. The three parties would engage most frequently in information exchanges during the phases of construction and operation and as a result, the interfaces for these operations have become relatively complex. Any flaws or oversight at any stage of operation would result in increased operational cost later on. Not only that, once the construction crew have left the site upon project completion, the lack of reference and data for the identification of liabilities could be a potential source of problem in the future. [2]

\subsection{Issues Pertaining System Encoding}

It is worth noting that the Revit software used for the purpose of this research does not contain formats that are compliant with the coding scheme adopted by the Public Construction Commission in its material database and 
category database. If the master format established by the Public Construction Commission were to be included in the system database for the BIM, data such as specifications, materials, quantities and cost estimates must be specified at the design phase to make so as to make the system more comprehensive. Major items, secondary items, minor items and unit prices in the master format of public constructions have been compiled and imported to the featured BIM system for relevant queries. For the purpose of this research, a text file containing the keynotes needed for this study has been created and stored in the Autodesk Revit database and associated with the software.

\section{SYSTEM ANALYSIS}

\subsection{System Function Analysis}

The "Electronic Building Construction Resume System" created in this research can be functionally divided into three major components, namely: "Basic data operation", "Shared Data Operation" and "Project Management Operation". The architecture of the system is shown in Fig 1.

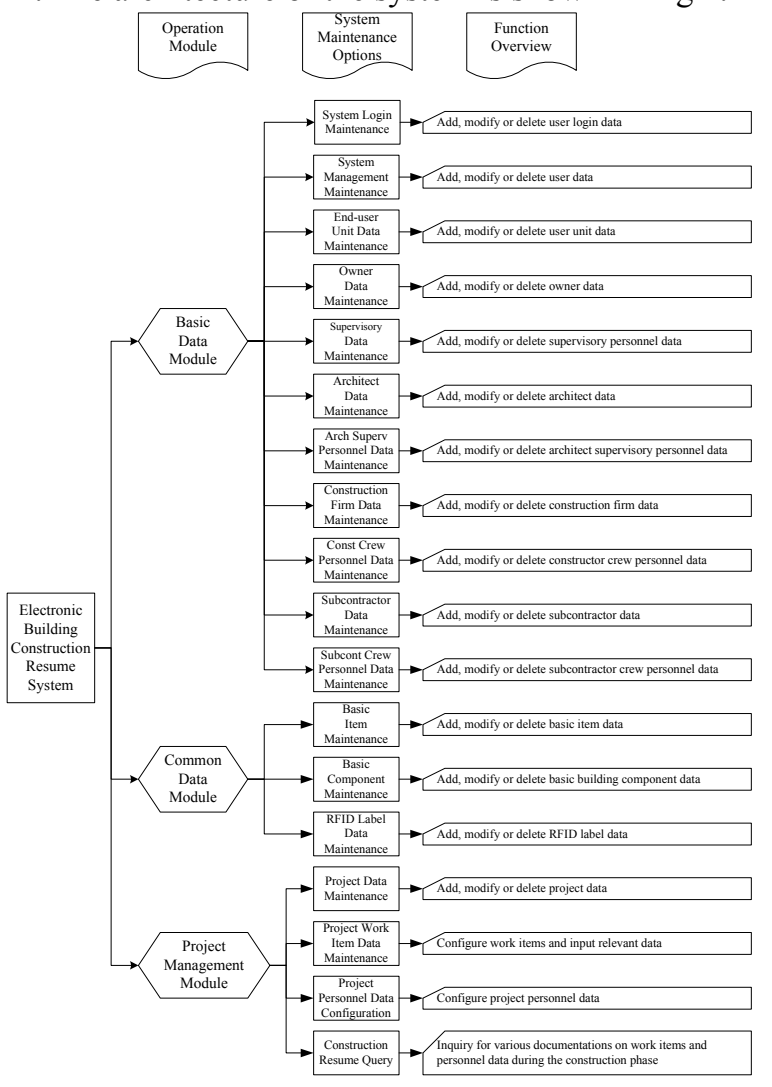

Fig 1. System architecture diagram

\subsection{Creation of Database Architecture}

In this research, ER/Studio has been used for the creation of E-R Model in conjunction with the BIM development tool Revit Architecture to import various parameter values and unit data table (created from receipts) into the database system using ODBC (as shown in Fig. 2).

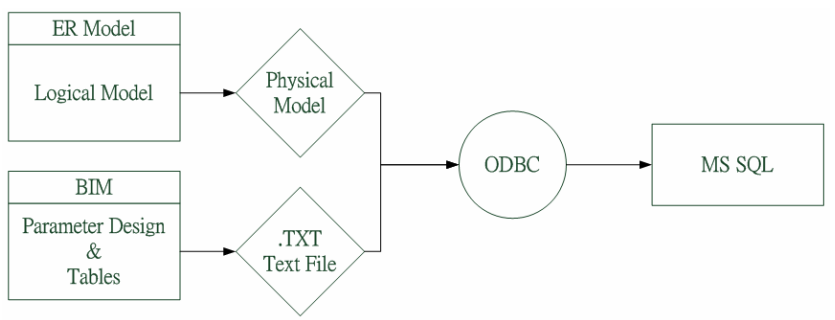

Fig 2. Illustration of data conversion procedure

The featured BIM is essentially a "joint database"; all 3D models created using the BIM are constructed from the data in the corresponding attribute tables, which are interrelated. This explains why changes to data would be propagated to other related data in real time for users to easily extract the required data. Both Revit Architecture and Archicad are mediums for the creation of BIMs and when used with other software and technologies in conjunction, these tools would ensure optimal efficiency for database use [3].

There are two type of database conversions presented in this research: one involved the use of ER-Model for the conversion of logic module to physical module before data is transferred from ODBC to MS SQL and the other would be Revit. Data conversion processes include data table conversion and conversion to relational database. Fig 3 below is a screenshot of data from Revit's internal database in SQL.

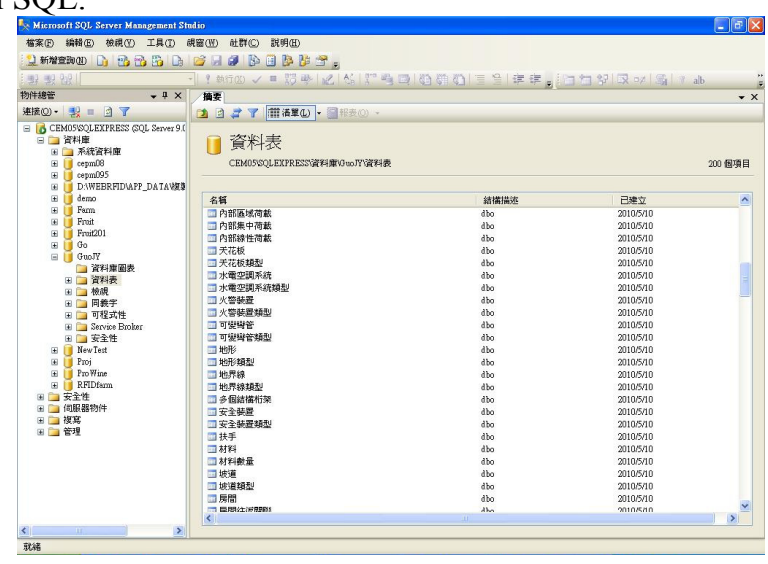

Fig 3. Data retrieved from Revit's internal data into SQL

\section{SYSTEM OPERATION AND VALIDATION}




\subsection{User Interface of the Management System}

The main menu of the system comprises of three items: "Basic Data Operation", "Common Data Operation" and "Project Management Operation":

\section{A. Basic Data Operation}

Basic data operation encompasses the maintenance of basic data such as the addition, deletion and modification of data. It includes "System Login Screen", "System Management Maintenance", “End-user Unit Data Maintenance”, “Owner Data Maintenance”, "Owner Supervision Data Maintenance”, “Architect Data Maintenance”, “Architect Supervision Data Maintenance", "Construction Firm Data Maintenance", "Construction Firm Supervision Data Maintenance", "Subcontractor Data Maintenance", "Subcontractor Personnel Data Maintenance" and so forth.

\section{B. Common Data Operation}

Common data operation covers modules such as "Basic Work Item Maintenance", "Basic Component Maintenance" and "RFID Label Data Maintenance". "Basic Work Item Maintenance" is related to the maintenance of basic system work items and it is further divided into major items, secondary items and minor items as shown in Fig 4 below.

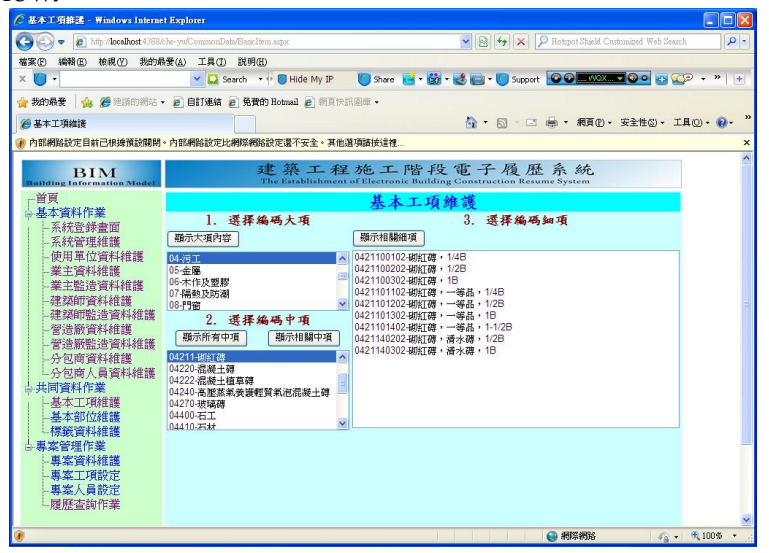

Fig 4. Basic construction item maintenance display

\section{Project Management Operation}

Project management operation encompasses the following functions:

1. "Project Data Maintenance": user can setup related information of a project in this function.

2. "Project Work Item Data Maintenance": users can use this function to configure various project work item settings and relevant documentations. Fig 5 is a screenshot of the data uploading screen.

3. "Project Personnel Data Configuration": users can use this function to configure the data for personnel related to the project work items. This includes supervisory personnel hired by the proprietor, architect and construction firm in addition to the construction crew hired by the subcontractors. Fig 6 is a screenshot of the configuration window.

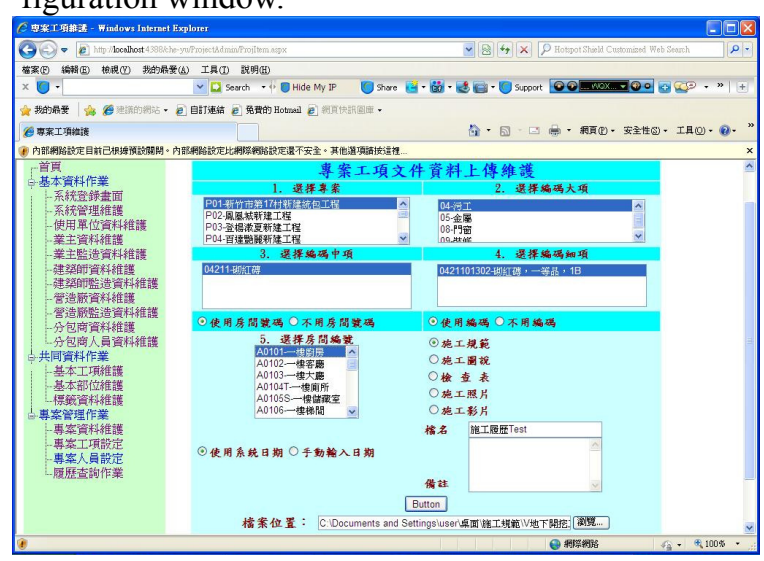

Fig 5. Documentation upload display of project work item

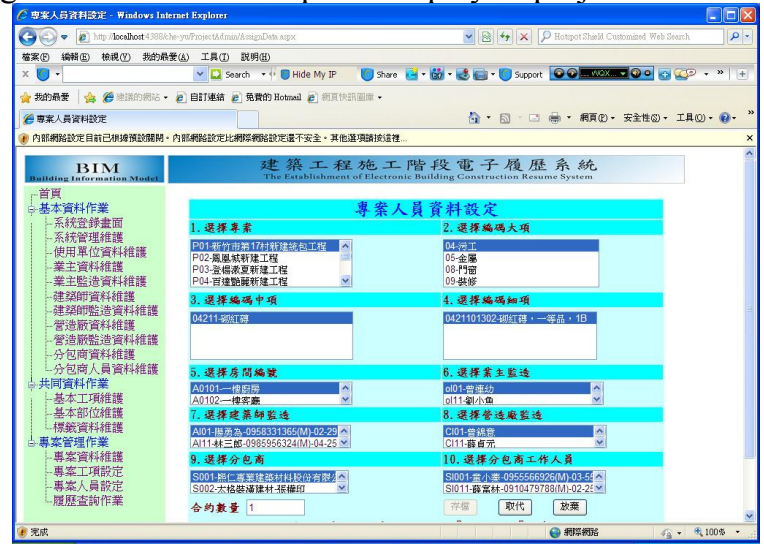

Fig 6. Project personnel data configuration display

4. "Construction Resume Query": users can use this function to search for relevant construction resume and download the file by clicking its link as shown in Fig 7.

The featured system uses SQL syntax to input relevant data into the system as text files. The data that was imported to the system for validation and debugging purposes is shown in Table 1. 


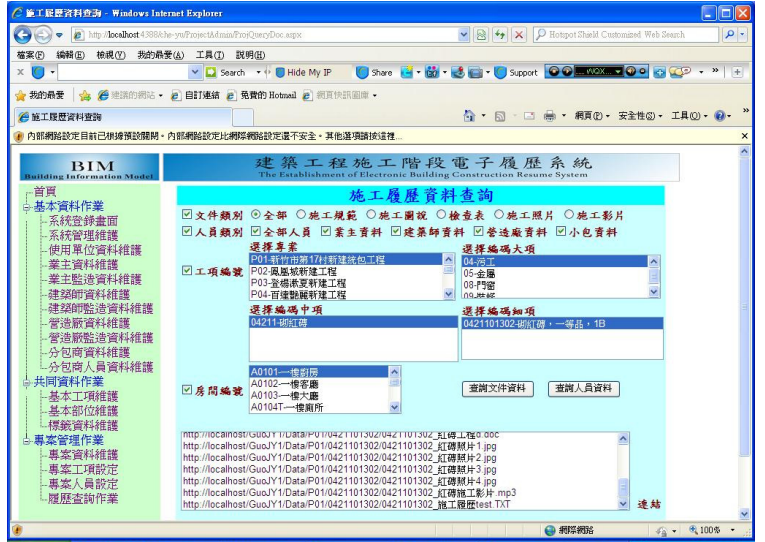

Fig 7. Resume documentation download display

Table 1. Summary of data imported into the database

\begin{tabular}{|l|l|}
\hline Data & Amount \\
\hline Public Construction Commission Format & 11,033 \\
\hline Personnel Data & 339 \\
\hline Document Files & 336 \\
\hline Total & 11,708 \\
\hline
\end{tabular}

\subsection{BIM System Linkage}

In this research, the parametric design feature of BIM has been linked to the "Electronic Building Construction Resume System" in order for relevant data to be directly configured in the system on the BIM platform. Figures 8 through 11 depict the model that was created for this research in the BIM.

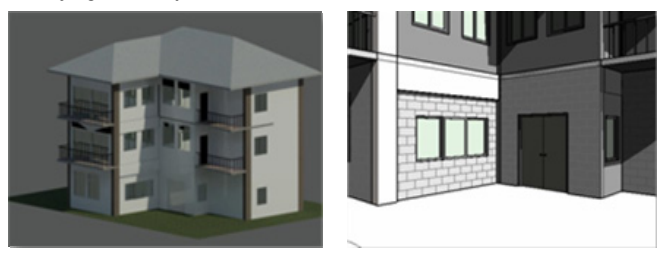

Fig 8. Project perspective created using the BIM
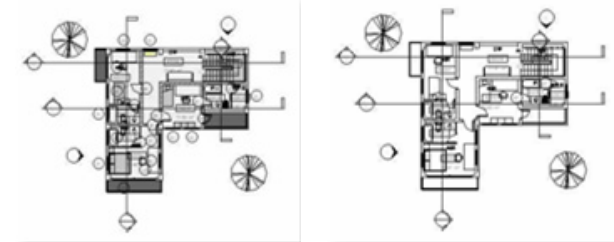

Fig 9. Project floor plan created using the BIM

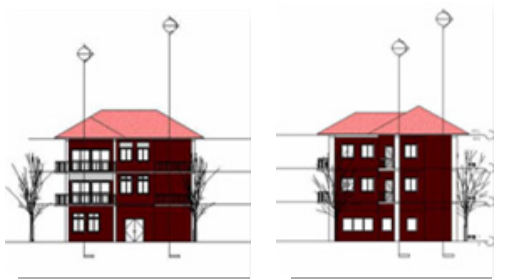

Fig 10. Project floor plan created using the BIM

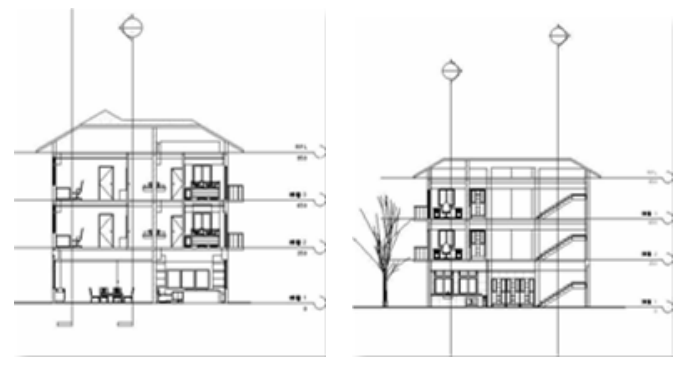

Fig 11. Project cross-sectional drawing created from BIM All structures created using Revit are based on the objects at the corresponding parts. This means the user can configure the selected part directly. Fig 12 shows the selection of "Element Attribute" and the configuration to associate it to the system.
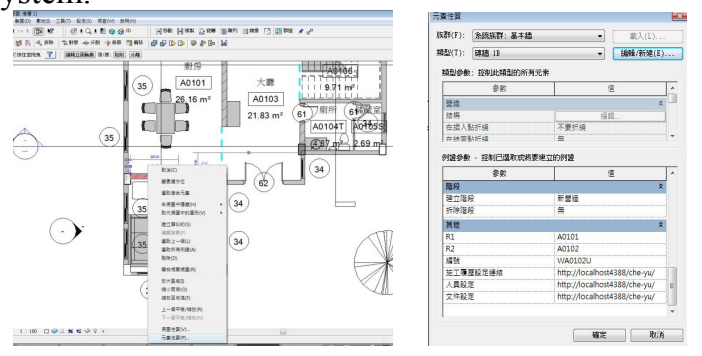

Fig 12. Screenshot of construction resume configuration association in Revit Architecture

\subsection{System Features}

The "Electronic Building Construction Resume System" created for the purpose of this study has the following features:

\section{A. Anticipatory data input}

All the essential data have been keyed into the system in advance with most work items codified according to the master format established by the Public Construction Commission so as to accommodate to the needs of the construction industry.

B. Easy data configuration

Items such as construction firms, room numbers and so forth created using the system are complete with dropdown query function along with automatic system association functionality. In addition, the user can also access all relevant sub-items by clicking the items in the Public Construction Commission format.

C. Comprehensive association and integration for all data All work item related documentations are accessible to the user through the query function, which allows the 
user to search for all documents or for documents under specific category. The user can also search for project personnel data that has been previously established in the system by specifying the work item or room number. In addition, the user can also search for supervisory/construction personnel in conjunction or independently.

D. Incorporation of RFID technologies for identification When the RFID Reader picks up signals from room labels, the system would be able to access relevant information on the room, including relevant documentations and personnel files as reference for actions to be taken.

E. Integration with BIM

Links are first configured in Revit Architecture in order to convert the data needed in the preparatory stage of construction for storage in the system database to be used in conjunction with relevant documentations in the construction phase and personnel data.

F. Failsafe measures

The RFID labels are designed with failsafe features and uniquely assigned to specific room numbers. Once in use, a label would be marked accordingly to prevent the issue of repetitive use.

G. Easy inquiry on a web-based interface

The featured system is designed to have a web-based interface to allow users to search for relevant information at anytime, anywhere over the internet with a browser to ensure optimal utilization.

\section{CONCLUSION}

Through the review of local and foreign literatures, the study presents the application of BIM at different stages of building lifecycle along with the development of "Electronic Building Construction Resume System", which includes modules such as "Basic Data Operation", "Common Data Operation" and "Project Management Operation". After the completion of system development, relevant data was imported to the system to ensure that the system was able to fulfill all relevant procedures and functional requirements. With numerous tests and minor modifications to the database structure, the system has been significantly improved to deliver the information and data that users may need to ensure full preservation of data from the construction phase. The following is a summary of the conclusion for this research:

1. Relevant research and studies have proposed relevant theories on the preservation of building lifecycle data. However, a comprehensive study on the methods and steps of preserving building lifecycle data from the concept of BIM has yet to be presented.

2. The inquiry interface designed for the featured system was specifically created to allow the user to create links to documentations from construction phase and search for personnel data to overcome the inconveniences of data inquiries by offering better presentation and accessibility to documentation and personnel data. The use of RFID technology with labels as carriers of relevant information for rooms in a building also helps to shorter search time and improves the timeeffectiveness of document use.

3. The application of the featured system would deliver better quality in terms of usage maintenance while facilitating the promotion of IT adoption for the construction industry by easing the process of data inquiry to prevent the inconveniences caused by data loss.

\section{REFERENCE}

[1] Tu Shih-Chung, Tsai Meng-Han, Introduction to BIM tools, Construction News Record, 321, Page 47-54, 2009.

[2] Hsieh Shang-Hsien, Standardized information technology for the realization of building information management, Proceedings of the Conference on Construction CALS, Page 31-47, 2008.

[3] Tu Shih-Chung, Tsai Meng-Han, Technological development and process of BIM, Construction News Record, 316, Page 60-67, 2009. 